\title{
THE TREATMENT OF HYPERTENSION
}

\author{
By G. S. C. SowRY, M.D., M.R.C.P. \\ Assistant Physician, Edgware General Hospital, Middlesex
}

\section{Hypertension and Cardiovascular Disease}

Amongst the adult population of modern societies, cardiovascular disease is probably the major cause of death. To a large extent it is inseparable from the process of ageing and therefore must remain an integral part of life. When it strikes prematurely, however, it becomes a tragedy for which medicine must strive to find a remedy.

The factors responsible for cardiovascular disease are numerous. An unduly large proportion of such disease is probably ascribed to hypertension and hypertension has become a convenient aetiological pigeon hole for degenerative heart disease. 'Hypertensive heart disease' is thus often diagnosed in elderly patients with heart failure, an enlarged left ventricle and arterial pressure of, say, $160 / 90 \mathrm{~mm}$. Hg. And yet in a study of the arterial pressure of a random sample of the population, the author and his colleagues found such pressures to be the average in women aged 60 (Hamilton, Pickering, Roberts and Sowry 1954). Because of a positive skewness of the distribution curves, more people of this age had pressures below these figures than above; but some 40 per cent. had pressures in excess of this. Now these persons were not in heart failure, nor had they manifest heart disease. It is pertinent to enquire, therefore, why a physical state which exists in 40 per cent. of the population should be held to be the main aetiological factor for a disease which is present in only a small fraction of their numbers. The danger of such a diagnosis is twofold: firstly, and of immediate concern to the patient, it may lead to treatment directed against what is in reality only a minor aetiological factor. And secondly, it engenders a state of pathological selfsatisfaction wherein no search for further aetiological factors is required.

This confusion of hypertension with cardiovascular disease is revealed in other ways. It happens occasionally that evidence is sought as to the incidence of hypertension in a group of persons not available for examination. Thus Platt (1947) when investigating the inheritance of hypertension accepted histories of cardiovascular disease in $\vec{\omega}$ relatives of the subject studied as evidence of $\frac{}{0}$ hypertension in these relatives. Sobye (1948) does likewise in a similar investigation, although perhaps with more justification since he limits himself to those relatives who had cerebral vascular $\omega$ accidents. Hypertension is common in such cases of ' stroke' but is by no means inevitably present; whilst if other vascular accidents (e.g. coronary thrombosis) are considered the incidence is lower still. Thus Masters (1953) using his new criteria $z$ of hypertension finds only 27 per cent. of men? with coronary thrombosis had pre-existing hyper- $\frac{\Phi}{3}$ tension. The most surprising example of the identification of hypertension with cardiovasculgr $\stackrel{\mathbb{S}}{-}$ disease is provided by Evans (1948) who demand $\overrightarrow{0}$ the presence of the latter before permitting tes diagnosis of the former.

The association between hypertension and cardiovascular disease is complex. The arteriolar necroses found in hypertension in the malignanto phase appear to be directly related to the height $\bar{\Phi}$ of the pressure, and especially of the diastolic pressure (Pickering, 1952). Once a level, critical for each individual, is exceeded then the cycle of events which is recognised as malignant hypertension supervenes. Other pathological changes are less directly connected with hypertension. Atheroma is more common in hypertensive than in normal subjects, and the causal relationship of 3 . the hypertension is shown by the development of atheroma in the pulmonary arterial tree in such conditions as mitral stenosis where pulmonary hypertension exists. But atheroma is found fre-? quently in the absence of hypertension. Cardiac failure, where there is no valvular or ischaemic cause, is common in hypertension; but similar casese are seen, although less commonly, where hyper- $N$ tension is, and as far as is known has been, absent. N The finding of left ventricular hypertrophy in such ${ }^{\omega}$ cases lends support to the supposition of preexisting hypertension but there is evidence that the cardiac failure itself may be responsible for the hypertrophy. The relationship, therefore, is largely indirect and the most that can be said is 
that certain types of cardiovascular disease are more frequently found in subjects with high pressures.

Robinson and Brucer (1939) claim that there are, broadly speaking, critical levels of systolic and diastolic pressures above which expectation of life begins to fall away. As a result they deduce that pressures above $140 \mathrm{~mm}$. Hg. systolic and $80 \mathrm{~mm}$. $\mathrm{Hg}$. diastolic are suspect, regardless of age; whereas 150 and $90 \mathrm{~mm}$. $\mathrm{Hg}$. represent the lower limits of pathological hypertension. Closer examination of their figures shows that the rise of mortality with increasing pressures is evident at all levels of pressure but that, the relation being curvilinear, the rate of increase in mortality is greater above these pressures than below. There is, in fact, no abrupt change in prognosis at any level of pressure, other than that which is associated with the development of malignant hypertension. None the less, within certain limits, the higher the pressure the less the expectation of life.

Hypertension is thus given pride of place in the causes of cardiovascular disease. No one will contest that it is a factor of great importance but it is essential to maintain a sense of proportion, to realise that such disease can and does occur in the absence of hypertension and, of greater importance, to appreciate fully that hypertension can and does exist without cardiovascular disease. For it is seldom hypertension itself that is disabling but the cardiovascular disease which may accompany it.

\section{The General Indications for Treatment of Hypertension}

Hypertension may necessitate treatment either by reason of the gravity of the prognosis or by virtue of the severity of its symptoms. Where the prognosis is undoubtedly bad, then treatment is an urgent matter. Evidence of the malignant phase of hypertension is associated with a course which is almost always progressively downhill to death in a year or less. At the present time this is perhaps the strongest reason for treatment directed to lowering the arterial pressure. Such a diagnosis results from the presence of papilloedema, retinal haemorrhages and soft exudates (Grade IV of Keith, Wagener and Barker , 1939). Commonly there is albuminuria and a diastolic pressure of $140 \mathrm{~mm}$. $\mathrm{Hg}$. or more, but neither of these latter findings is essential to the diagnosis. The prognosis is less certain, but still unfavourable, when soft exudates and haemorrhages exist in the absence of papilloedema. Moreover, there is often doubt as to the presence or absence of the papilloedema and in such cases the need for treatment is less clear cut, although it will be advisable in many.

Patients with left ventricular failure have a poor expectation of life, and where existing hypertension can be held to be the major cause for such failure, treatment of the hypertension is required in the absence of definite contra-indications. Leishman (1953) drew attention to the presence of cardiac enlargement and electror cardiographic changes in patients whose hypertension ran an adverse course. The presence of these two signs therefore enhances the need for active treatment.

The height of the pressure is of considerable actuarial importance, but to particularise from statistical experience is often unrewarding, and it is by no means rare to observe for many years the continuing good health of subjects with diastolic pressures above what are commonly considered danger levels. For individuals, therefore, the height of the arterial pressure is of limited value in prognosis. It follows that the arterial pressure itself cannot be used as a definite indication for treatment. One can perhaps set a definite lower limit of arterial pressure below which treatment is not indicated and a diastolic pressure of I $10 \mathrm{~mm}$. Hg., chosen by Evans (1948) as the lower limit of hypertension, is perhaps convenient in this respect.

Treatment may be required for the relief of symptoms but, just as it is essential to be able to ascribe pathological vascular disease mainly to hypertension before undertaking the treatment of the latter, so it is necessary to be certain that the symptoms under consideration are due to the hypertension. If hypertension is a common cause of symptoms, as common a cause is the anxiety induced by the knowledge of hypertension (Stewart 1953). Certain features enable a distinction to be made between the symptoms due to hypertension and those of an accompanying anxiety state. Headache, common to both, is classically occipital in the former, occurring maximally on waking and diminishing with activity: in anxiety neurosis it is often vertical, worse towards the end of the day and of a constricting or pressing character. Dyspnoea and palpitation are more difficult to ascribe with certainty: when due to the hypertension itself there is usually accompanying evidence of left ventricular hypertrophy or failure. Relief of vertigo by medical treatment of hypertension has been claimed (Morrison, 1953). Age is of importance in assessing the need to lower arterial pressure. The expectation of life is probably greater in a young hypertensive subject than in an elderly patient with the same arterial pressure, but the difference from the normal expectation is of course far greater in the young subject. The 
need for treatment is thus greater; furthermore, the results of treatment are better in the comparatively young. No fixed age limit can be set beyond which radical treatment should not be undertaken, but senility is an obvious contraindication.

The comparatively young subject who has had a cerebral or coronary thrombosis and who has severe hypertension is a therapeutic problem. Medical and surgical measures to lower the pressure have been given their trial, but the risk of further thrombosis is increased by such treatment, and it is generally accepted that a past history of such vascular thromboses is a contraindication to any treatment directed to radical reduction of arterial pressure. In such patients it is worth while to consider whether there is a place for the most severe restriction of fat intake in an attempt to limit and perhaps even to reverse the development of atheroma. No reliable results are available as yet but the matter has been reviewed extensively by Keys (1952). Subarachnoid haemorrhage when associated with preexisting hypertension needs special consideration. A raised arterial pressure may result from the intracranial haemorrhage itself and in patients first seen with such a condition no immediate treatment to lower the arterial pressure is required. Where hypertension is known to have preceded the haemorrhage, medical treatment to lower the pressure is worth undertaking, as it is when observation of a new patient shows the arterial hypertension to be persistent. This is particularly so since the results of vascular surgery in hypertensive subjects with subarachnoid haemorrhage are not so encouraging as when there is no hypertension.

The majority of hypertension over the age of 40 years is of the essential variety. Below this age in many cases renal causes exist although the demonstration of such a cause in life is often difficult, and at times impossible. Where such a cause can be found, and when as in some cases of chronic pyelonephritis the disease can be shown to be unilateral, nephrectomy results in a reduction of pressure in a proportion of cases sufficient to encourage continuance of this operation (Pickering and Heptinstall, 1953). Where the disease is bilateral, medical treatment of the hypertension is not contra-indicated and may bring about considerable improvement in the patient's condition (Morrison, 1953) unless there is evidence of serious renal failure, when treatment is probably not advisable.

Other secondary forms of hypertension, such as Cushing's syndrome, phaechromocytoma and coarctation of the aorta all have their appropriate methods of treatment and must be borne in mind.
It is worth noting that a considerable number of such cases of secondary hypertension remain with $\frac{3}{8}$ an appreciable, if diminished, hypertension after $\stackrel{\circ}{\circ}$ the successful extirpation of the underlying cause, $\stackrel{\leftarrow}{\leftarrow}$ an observation which perhaps throws some light upon the pathogenesis of sustained hypertensionof whatever aetiology (Hamilton, Litchfield, Peart등 and Sowry, 1953; Smirk, 1949), and which has $\overline{\bar{n}}$. some bearing upon the theoretical value of the intermittent reduction of arterial pressure which $\stackrel{\circ}{\circ}$ may result from medical treatment.

\section{Treatment Available}

Hypertension is a chronic condition and it is $\vec{\sigma}$ important to be on a secure therapeutic basiso before commencing treatment. It must always $\frac{\varrho}{3}$ be questioned whether the treatment to be given is likely to do good, and if so, whether this goodo will exceed the harm likely to ensue. Until some $\omega$ simple, safe, reliable and innocuous method of ${ }^{\circ}$ lowering arterial pressure is found, it must be realized that the improvement that can be bestowed $\mathrm{O}$ upon the mild hypertensive is limited. In many mild cases of hypertension, the wisest course is to $z$ say nothing to the patients of their hypertension; if $O$ they are already aware of it, reassurance is prob- $\frac{\Phi}{3}$ ably all that is required; a reassurance based the knowledge that most persons show pressurgs that rise with age and that many hypertensive $\overrightarrow{0}$ subjects live to a good age in health. It is in th type of patient that a more realistic approach needed. Too often are they given sedatives and told to restrict their activities, thus being impregnated with the germ of invalidism. If such treatment and advice were known to be of benefit, $\frac{\circ}{D}$ there would remain the question of whether the benefit exceeds the harm caused. It is probable $\stackrel{0}{\partial}$ that in many cases the phenobarbitone merely depresses, and that the inactivity only demoralizes. The lowering of pressure which may result is of little prognostic importance and indeed the game is scarcely worth the candle. If apprehension is to be avoided in such patients, the advice must, where possible, be bold and uncompromising; no restriction should be imposed

This approach is, of course, not possible where there are symptoms of any magnitude and the patient with only a moderate hypertension, but $\frac{D}{O}$ with considerable resultant headache, presents a problem to which there is no simple answer. In o this type of patient some authorities (d'Abreu, $N$ 1953) claim good results from sympathectomy, $N$ but it is felt by others (McMichael, I952) that the benefits from such extensive operations are too unpredictable, not occurring at all in some patients $\stackrel{\circ}{\subset}$ and not sustained in others. Headache may be $\stackrel{\varnothing}{\mathscr{N}}$ relieved by sympathectomy even when the arterial pressure is not reduced (Platt and Stanbury, I950), 
but this is infrequent and it is the author's experience that symptoms return eventually in such apparent successes. It is relevant to note that headache in hypertension commonly disappears rapidly when the patient is confined to bed for any reason, not to return for a period far exceeding the fall of the blood pressure. The results of sympathectomy are, in general, disappointing and other lines of treatment must be considered. The use of hexamethonium or related compounds has much to recommend it in such cases, although it will be a matter for debate as to whether the relief obtained warrants the inconvenience and discomfort caused. The pitfall in such therapy in cases of no great severity is that the treatment is often not given by the physician with the necessary enthusiasm. Inadequate treatment results and the symptoms remain. If it is decided, perhaps after a trial, that hexamethonium therapy is not worth while, then any factors which may adversely affect the prognosis remain to be considered and treated. Particular attention, where applicable, must be paid to weight reduction, for not only does this reduce the arterial pressure, but the liability to cardiovascular disease is also diminished.

When the signs of malignant hypertension exist, there can be no doubt that radical treatment to lower the arterial pressure is called for. Sympathectomy here had its gireatest place, but such subjects were poor operative risks and the mortality was high; furthermore, the results were once again often disappointing. But until the introduction of the methonium compounds sympathectomy was the best that could be offered. Since then, however, opinion has swung towards treatment with medical agents to lower the pressure. No patient is too sick to be given the benefit of such treatment and previous contraindications to surgery, such as a raised blood urea or a failing heart, are no objection to medical treatment. Hexamethonium can with safety be given to patients in left ventricular failure with good results. Methonium compounds may produce an improvement in incipient renal failure, although occasionally the blood urea will rise more steeply, when treatment must be discontinued. In the presence of renal failure, excretion of methonium compounds is delayed, with a consequent prolongation of action and the risk of cumulative effects. Such patients require smaller doses than normal, or the interval between doses may be lengthened.

Other drugs have been used in an attempt to lower blood pressures. Thiocyanates proved unsatisfactory by virtue of toxic effects and difficulty of control. Veratrum alkaloids have their advocates (Kauntze and Trounce, I95I), but most patients find the toxic side-effects too severe to allow continued treatment. Experience in this气 country with the alkaloids of rauwolfia serpentin氶 is inadequate to allow accurate assessment of theip worth. Arterial pressure can be reduced by. extreme reduction of salt intake, but this must not exceed $25^{\circ} \mathrm{mg}$. per day (M.R.C., I950). Continued existence upon a diet as restricted as this is well-nigh impossible.

It appears, therefore, that at the present timed hexamethonium or related compounds offer the most satisfactory method of reducing raised? arterial pressures, except in the rare and fortunate $\overrightarrow{0}$ cases where a primary cause for the hypertension can be found and removed.

\section{Treatment with Hexamethonium and Relate Substances}

It is not proposed to give here a detailed accounf of methods of treatment nor to list all the prepara $A$ tions which have been used. It will be sufficient to note the substances in common use, theiffo methods of administration and of control and theo difficulties which may be encountered.

Hexamethonium bromide has been used more $z$ extensively than other compounds. It is available in ampoules or multi-dose bottles for subcutaneous injection and in tablet form for oral administration A solution in polyvidone solution to prologg absorption is also marketed. A similar substaneeco again for oral or subcutaneous administrati exists in the form of the bitartrate of her methonium: this was introduced to avoid thes symptoms and signs of bromism noted in some patients receiving large oral doses of the bromide् salt. The use of this compound has becomed unnecessary because of the abandoning of ora? therapy and consequent reduction in total dosage? More recently still, pentapyrrolidinium bitartratê ('Ansolysen ') has been introduced, and is more potent and longer acting with, it is claimed, less. side effects (Wien and Mason, r953; Maxwell and Campbell, 1953; Smirk, I953). It can be given. orally or subcutaneously, and a retard solution exists in polyvidone solution with ephedrine 0.5 per cent.

These substances act by blocking the transmission of impulses through autonomic ganglia? They are excreted by glomerula filtration and the effects produced are believed to be proportiona to the plasma concentration (Morrison and Patonfs I953). Ideally a preparation which could bه administered in such a way as to produce a con stant and readily controlled plasma concentration is required. Oral administration of hexamethos nium salts proved most unsatisfactory; absorptiof was too erratic and the margin between treatment? that was on the one hand ineffective but safe and that which was effective but precarious prove $\$$ 
too narrow. Parenteral treatment has largely replaced it, the patients giving their own injections, as do diabetics. Subcutaneous hexamethonium bromide requires 8-hourly injection to produce a satisfactory lowering of pressure. Even with such frequent injections, the pressure may well have risen to near pre-treatment levels two or three hours before each injection, but it is probable that there is appreciable gain from even intermittent lowering of pressure. With all these compounds the response to the first exceeds that to subsequent similar doses. Providing, therefore, that no alarming symptoms occur after the initial trial dose and that the closely watched arterial pressure does not fall too low, it is safe to commence regular treatment with a higher dose, thereafter rising by suitable increments until a satisfactory level is reached. Tolerance is acquired at varying rates by different subjects, and whereas one patient may become stabilized within a week, another may require increasing amounts over a much longer period.

In assessing the correct dose, use must be made of the pronounced fall in pressure which results on standing. A patient stabilized whilst in bed will be found to be receiving too much of the drug when up and about. Therapeutic use should be made of this postural fall in pressure and all patients should get up as early as possible; if not fully ambulant, they should sit in a chair during the day. The aim should be to reduce the arterial pressure after such injection for as long a period and to as low a pressure as the patient can safely tolerate without severe side effects. Trial and error is the only method of assessing the patient's requirements and during the initial period of stabilization, a careful observation of blood pressure, lying and standing, before and after injections, is necessary. Even with this, anxiety is often felt when the time comes to allow such patients to leave hospital.

The degree of care exercised in the control of out-patients must vary; it will be possible elaborately to organize some clinics, along the lines of a diabetic clinic, with frequent attendances by the patients and with technicians to record repeated pressures in each subject. In others a more humble organization may be the best that can be offered. In all, it is essential that readings of blood pressure, in the lying and standing position before and about three-quarters to one hour after injection should be taken from time to time. Where, as is most usual, such treatment is being controlled from hospital, it is obviously of great importance that the patients' general practitioners should be aware of the treatment being given, so that immediate action can be taken, as in a diabetic, if treatment proves excessive. The parallel with diabetes is close, since variations in requirements may occur for no apparent reason and intercurrent infections may greatly increase the effect of the drug (unlike in diabetes, where infections raise the insulin requirements). There is a place therefore for a card, to be carried by the patient, showing the dose of the substanceibeing given, as is now the custom in many diabetic clinics. Since most patients receive stocks dispensed so that their dose is in a unit of measurement (e.g. $75 \mathrm{mg}$. in I ml.), such a card would overcome the difficulty occasioned by the patient merely knowing the volume of the injection.

The choice of the preparation used must be a matter for individual decision. Although not having had as much experience with 'Ansolysen' as with hexamethonium bromide, it is the author's opinion that the former, by subcutaneous injection, and particularly in the retard preparation, offers the most satisfactory treatment at present available. The recommended initial dose of the aqueous preparation is $3 \mathrm{mg}$. and the dosage increment $\frac{1}{2}$ to $1 \mathrm{mg}$., whereas $4 \mathrm{mg}$. of the retard preparation is recommended initially, with increments of $\frac{1}{2}$ to $1 \frac{1}{2} \mathrm{mg}$. With both preparations, 12-hourly injections need to be given. Much larger doses are required for oral administration and $20 \mathrm{mg}$. is recommended initially, with increases of a further $20 \mathrm{mg}$. The author has only limited experience of the oral method of treatment with 'Ansolysen,' but this experience is not especially encouraging.

The dose of hexamethonium bromide is considerably greater. The dangers attendant upon oral therapy, with its unexpected and sudden variations in absorption, precludes this method of administration. Subcutaneous injection needs to be repeated every 8 hours and $25 \mathrm{mg}$. is an appropriate trial dose. If the effect is moderate it is safe thereafter to continue with $50 \mathrm{mg}$. injections 8-hourly; as tolerance develops increments of $\mathrm{I} 5$ to $25 \mathrm{mg}$. are made until a satisfactory and stable dose results. Despite close observation of patients under standard conditions, unexpected variations in requirements occur from time to time.

\section{Side-effects}

Some degree of postural hypotensive symptoms during the first hour after each injection are to be expected in a successfully treated patient. If treatment during the remainder of the interval between injections is to be of any effect, these early symptoms must be endured and the patient must regulate his life to avoid standing during this time. Loss of accommodation is of annoyance but not incapacitating, and it is claimed that relief of this symptom, as of dryness of the mouth and of 
micturition difficulties, can be achieved with 25 to $50 \mathrm{mg}$. of ' mecothone' sublingually with each injection (Morrison, 1953). Severe intestinal stasis, as was commonly encountered in oral hexamethonium therapy, is not a problem of magnitude with subcutaneous therapy and bromism does not occur with the small dosage of hexamethonium bromide required parenterally.

Despite the surmountable nature of all the side effects, the labour and courage required to continue with regular injections for an indefinite period are considerable. The successful conquest of the side effects requires an intelligent and persevering character in the patient. A weak intelligence or a low morale may render treatment along the lines suggested quite impossible.

\section{Duration of Treatment}

In the present state of knowledge, it appears that treatment may well have to be continued for an indefinite period. No doubt it will be stopped in some cases where the disease has taken a favourable course, and such cases will continue to fare well. But it would be premature to hope for this in all cases, although there are theoretical grounds for believing that the necrotizing arteriolitis of malignant hypertension may be arrested and the vicious circle broken if arterial pressure can be reduced even temporarily.

\section{Summary and Conclusion}

I. The relationship between cardiovascular disease and hypertension is discussed. The false identification of the two conditions is emphasized, and the benign nature of much of hypertension is stressed.

2. The indications for treatment aimed at reducing arterial pressure are the gravity of the prognosis and, less commonly, the severity of the symptoms. Of the former, malignant hypertension is the most important: evidence of cardiac failure is an indication when hypertension is the main aetiological factor. The height of the blood pressure is not a reliable guide.

3. In attempting the relief of symptoms, care must be taken lest the symptoms induced by treatment exceed those relieved.
4. Age is no contra-indication but senility is.

5. Cerebral and coronary thromboses are contra indications to treatment. There is a place fo? pressure reduction by medical treatment in some cases of subarachnoid haemorrhage.

6. Renal hypertension is improved by medicat treatment in many cases.

7. The various methods available for reducing arterial pressure are discussed. A plea is madब for less sedation and for less restriction of activit in mildly hypertensive subjects.

8. The limitations and unpredictable results of $\vec{p}$ sympathectomy are discussed.

9. The most effective weapons at present avail able are hexamethonium and related compoundsg For general use pentapyrrolidinium bitartrate ('Ansolysen') in a retard solution is probably. the most reliable agent. The methods of ad ${ }_{\omega}$ ministration are described, together with side effects to be expected. No limit to the duration of treatment can be foreseen in most patients.

\section{BIBLIOGRAPHY}

D'ABREU, F. (1953), Lancet, i, 1164.

EVANS, W. (1948), ' Cardiology,' London.

HAMILTON, M., LITCHFIELD, J. W., PEART, W. S., an\$ SOWRY, G. S. C. (1953), Brit. Heart. F., 15, 241 . HAMILTON, M., PICKERING, G. W., ROBERTS, J. A. FФ and SOWRY, G. S. C. (1954), Clin. Sci., 13, 11.

KAUNTZE, R., and TROUNCE, J. (195I), Lancet, ii, 1002.

KEITH, N. M., WAGENER, H. P., and BARKER, N. (1939), Amer. F. Med. Sci., 197, 332. KEYS, A. (1952), ' Report to First International Congresड्.
Dietetics, Voedingsgebied, p. 92 .

LEISHMAN, A. W. D. (1953), Brit. med. F., i, 1131 .

MCMICHAEL, J. (1952), Ibid., 1, 933.

MASTERS, A. M. (1953), Circulation, 8, 170.

MAXWELL, R. D. H., and CAMPBELL, A. J. M. (1953), BriC med. F., $1,455$.

MEDICAL RESEARCH COUNCIL (1950), Lancet, ii, 509.

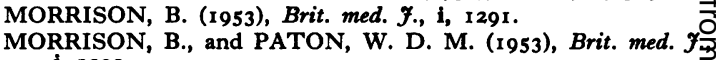
i, 1299 .

PICKERING, G. W. (1952), Circulation, 6, 599.

PICKERING, G. W., and HEPTINSTALL, R. H. (1953 Quart. $\mathcal{~}$. Med., 22, I.

PLATT, R. (1947), Ibid., 16, 111 .

PLATT, R., and STANBURY, S. W. (1950), Lancet, i, $65 \mathrm{I}$. ROBINSON, S. C., and BRUCER, M. (1939), Arch. Intern. Med'G 64, 409 .

SMIRK, F. H. (1949), Brit. med. F., i, 79r.

SMIRK, F. H. (1953), Ibid., i, 457.

SOBYE, P. (1948), "Heredity in Essential Hypertension and Nephrosclerosis,' op. dom. Biol. hered. hum.,Kbh., Vol. 16. STEWART, I. MC. D. G. (1953), Lancet, i, 1261.

WIEN, R., and MASON, D. F. J. (1953), Brit. med. F., i, 454. 